\title{
Prediction and Measurement of Initial Flocculation Rates in Quiescent Media
}

\author{
D. H. MELIK ${ }^{1}$ AND H. S. FOGLER ${ }^{2}$ \\ Department of Chemical Engineering. The University of Michigan, Ann Arbor, Michigan 48109
}

Received December 21, 1984; accepted April 9, 1985

\begin{abstract}
Initial flocculation rate experiments were carried out with a dilute suspension of bidisperse polystyrene spheres ( 0.166 and $0.255 \mu \mathrm{m}$ in radius) under normal gravity conditions and, using a centrifuge, under conditions of an enhanced gravitational flow field. These experiments were designed to study the effect of differential creaming between small and large particles on the flocculation process and also to ascertain some of the limitations associated with the DLVO theory of electrostatic stabilization. A turbidity technique which accounts for the simultaneous effects of flocculation and creaming was developed to determine the initial flocculation rate. By comparing theoretically predicted rates with experimentally observed ones for each monodisperse system under normal gravity, the Hamaker constant was determined to be $A=2.87 \pm 0.36 \times 10^{-14} \mathrm{erg}$ for a retarded attractive potential, and the surface potential was found to be $\Psi_{0}=-12.7 \pm 0.5 \mathrm{mV}$. The corresponding $\zeta$ potentials ranged from -19 to $-32 \mathrm{mV}$. However, the predicted stability factors for the bidisperse system were over 6 orders of magnitude greater than the measured values when the $\zeta$ potentials were substituted for $\boldsymbol{\Psi}_{0}$ in the chosen electrostatic repulsion model, whereas, correct stability predictions were made with the characteristic potential of $-12.7 \mathrm{mV}$. Under conditions of an enhanced gravitational flow field, the bidisperse flocculation rate increased with increasing gravitational force; in accordance with our previous theoretical predictions. For the larger gravitational forces studied, there appears to be a high degree of coupling between Brownian motion and gravitational forces. (c) 1985 Academic Press, Inc.
\end{abstract}

\section{INTRODUCTION}

In essentially all rigorous studies of creaming (1-3), Brownian flocculation (4-10), gravity-induced flocculation (11-13), as well as shear-induced flocculation (14-20, 79), the interparticle forces are described by the widely accepted DLVO theory $(21,22)$. Unfortunately, some of the physical parameters which enter into this theory have not been determined unambiguously. The Hamaker constant, which controls the magnitude of the van der Waals attractive force, can be computed from dielectric permittivity data using Lifshitz theory (23). However, agreement with measured values of the Hamaker constant is usually only within an order of magnitude. The

\footnotetext{
${ }^{1}$ Present address: The Procter \& Gamble Company, Miami Valley Laboratories, Cincinnati, Ohio 45247.

${ }^{2}$ To whom all correspondence should be sent.
}

electrophoretically determined $\zeta$ potential is commonly used as the characteristic potential in the calculation of the electrostatic repulsive force, although the relationship between the $\zeta$ potential and the surface potential remains unclear (24).

Brownian flocculation experiments with monodisperse systems have verified several of the qualitative features of the DLVO theory (25-27), and have yielded estimates of both the Hamaker constant $(18,28,29)$ and an effective surface potential $(25,27)$. Using shearinduced flocculation experiments, van de Ven and Mason (16) verified an interesting consequence for particles controlled by DLVO interactions when subjected to a shearing field. Under conditions of significant electrostatic repulsion, particles flocculate at low and high shear rates, but remain stable at intermediate shear rates $(15,17)$. An analogous phenome- 
non has also been predicted for gravity-induced flocculation (11). Zeichner and Schowalter (18) used shear experiments to estimate the Hamaker constant by comparing theoretical prediction with experimental observation. The resulting Hamaker constant was found to be in good agreement with the one they determined from rapid Brownian flocculation experiments.

There have also been Brownian flocculation studies with mixtures of two monodisperse suspensions of different sizes (30-36). These investigations yield valuable information on the relative behavior of small and large particles in hetergeneous systems. Unfortunately, with Brownian flocculation experiments, the heteroflocculation process is somewhat masked by the homoflocculation processes occurring simultaneously. Consequently, an overall stability factor is usually measured instead of the more crucial bidisperse stability factor. Therefore, when deviations are observed between theory and experiment, it is hard to ascertain which flocculation rate(s) are incorrect since there are three simultaneous processes occurring as a result of the three types of doublets possible with bidisperse systems. Another possibility is to have agreement between theory and experiment only because of compensating errors in computing each flocculation rate. In order to alleviate these potential problems, the actual bidisperse flocculation rate should be measured independently of the two homoflocculation rates. This problem is easily rectified with the turbidity technique proposed in this paper.

In another related study, Adler (19, 37-39) has extended the work of van de Ven and Mason $(15,16)$ and Zeichner and Schowalter $(17$, 18) by considering the effect of simple laminar shearing on different-sized spheres. The main emphasis of his experimental studies was to observe the relative trajectories of two unequal spheres under a variety of conditions. While there was good agreement between the theoretical and experimental trajectories, no measurements of the bidisperse shear-induced flocculation rate were made.
Based on our theoretical studies into the effects of gravitational forces on the flocculation process $(10,11)$, gravity-enhanced flocculation experiments appear to offer an advantageous means for studying the flocculation between different sized spheres. Increasing the gravitational flow field, such as achieved in a centrifuge, selectively increases the rate of heteroflocculation (as a result of the increased importance of gravity-induced flocculation), while not affecting the rate of homoflocculation (since homoflocculation is due solely to Brownian flocculation which is independent of the gravitational force). This effect demonstrates a distinct advantage of gravity-induced flocculation experiments over shear-induced flocculation experiments which tend to enhance the flocculation rate of the same sized particles as compared to the rate between different sized spheres (19).

Previous stability studies using enhanced gravitational forces on colloidal systems can be divided into two categories: (i) determining the separation kinetics of oil from oil-in-water emulsions by means of classical rate equations (40-43); or (ii) determining the gravitational force necessary to irreversibly flocculate particulate suspensions $(44,45)$. In each case, however, the colloidal system is totally creamed during the initial period required for the centrifuge to attain the preset speed. Consequently, the kinetics of the flocculation process are virtually impossible to study with their experimental techniques. The turbidity technique proposed in this paper will be shown to yield good estimates of the flocculation rate under conditions of an enhanced gravitational force field.

Initial rate experiments were performed to verify some of the effects of gravitational forces on the flocculation process described by Melik and Fogler $(10,11)$, and also to ascertain some of the limitations associated with the DLVO theory. The experiments consisted of first preparing and characterizing two monodisperse suspensions of spherical polystyrene particles. A turbidity technique was developed to measure the initial flocculation rates between dif- 
ferent sized particles undergoing simultaneous flocculation and creaming. Rapid Brownian experiments were performed on each monodisperse system to determine the composite Hamaker constant, and slow Brownian experiments were carried out to estimate the corresponding surface potentials. Using a bidisperse mixture of particles, initial flocculation experiments were carried out under normal gravity conditions and, using a centrifuge, under conditions of an enhanced gravitational flow field.

\section{INTERPARTICLE FORCES: DLVO THEORY}

Owing to the importance interparticle forces have in interpreting stability results, a presentation of the formulas used in the present study is warranted. The most commonly used interparticle potential model is described by the generally accepted DLVO theory $(21,22)$, which assumes that the total interparticle potential is obtained by summing the attractive and repulsive contributions, namely

$$
V_{\text {int }}=V_{\mathrm{A}}+V_{\mathrm{R}} \text {. }
$$

Although inadequacies of the classical DLVO theory are well documented $(18,23,46-49)$, there are many realistic situations under which the theory appears to be quite satisfactory $(16$, $18,50,51,79$ ).

The unretarded van der Waals attraction between spheres of radius $a_{1}$ and $a_{2}$ is given by Hamaker (52) as

$$
\begin{aligned}
V_{\mathrm{A}}= & -\frac{2 A a_{1} a_{1}}{3\left(a_{1}+a_{2}\right)^{2}}\left\{\frac{2}{R^{2}-4}\right. \\
& +\frac{2}{R^{2}-4(1-\lambda)^{2} /(1+\lambda)^{2}}+\frac{(1+\lambda)^{2}}{4 \lambda} \\
& \left.\times \ln \left(\frac{R^{2}-4}{R^{2}-4(1-\lambda)^{2} /(1+\lambda)^{2}}\right)\right\},
\end{aligned}
$$

where $A$ is the composite Hamaker constant, $R=2 r /\left(a_{1}+a_{2}\right)$ the dimensionless interparticle distance, $r$ the distance between particle centers, and $\lambda=a_{1} / a_{2}$ the particle size ratio. For retarded attractive potentials, the results of Ho and Higuchi (53) are applicable, namely

$$
V_{\mathrm{A}} \simeq-\frac{A a_{1} a_{2}}{3\left(a_{1}+a_{2}\right)^{2}(R-2)} Z\left(p_{0}\right)
$$

where

$$
\begin{aligned}
Z\left(p_{0}\right) & =\frac{1}{1+1.7692 p_{0}} \text { for } p_{0} \leqslant 1.0, \quad[4 \mathrm{a}] \\
& =\frac{2.45}{5 p_{0}}-\frac{2.17}{15 p_{0}^{2}}+\frac{0.59}{35 p_{0}^{3}} \quad \text { for } \quad p_{0}>1.0
\end{aligned}
$$

with $p_{0}=2 \pi(R-2) / \nu$, and $\nu=2 \lambda_{\mathbf{L}} /\left(a_{1}+a_{2}\right)$. The dimensionless parameter $\nu$ determines the degree of retardation where the characteristic London wavelength of the atoms, $\lambda_{\mathrm{L}}$, is typically $10^{-5} \mathrm{~cm}(54)$.

The electrostatic repulsion between unequally sized spheres under constant surface potential conditions is given by Hogg, Healy, and Fuerstenau (31):

$$
V_{\mathrm{R}}=\frac{\epsilon a_{1} a_{2} \Psi_{0}^{2}}{\left(a_{1}+a_{2}\right)} \ln \{1+\exp [-\kappa(R-2)]\}
$$

where $\kappa$ is the dimensionless reciprocal of the Debye-Hückel double layer thickness, $\epsilon$ the dielectric constant of the medium, and $\Psi_{0}$ the surface potential. The assumptions inherent in [2], [3], and [5] are well documented (11, $18,46,55,56)$

\section{EXPERIMENTAL METHODS}

\subsection{MATERIALS}

Two monodisperse polystyrene latexes, long considered the model colloidal system (57), were synthesized by the surfactant-free process of Goodwin et al. (58). The average particle size and polydispersity of each system was determined by both scanning electron microscopy and dynamic light scattering. Details are given in Table I. The original volume fraction

TABLE I

Details of Latex Dispersions

\begin{tabular}{ccc}
\hline $\begin{array}{c}\text { Mean radius } \\
(\mu \mathrm{m})\end{array}$ & $\begin{array}{c}\% \\
\text { Variation }\end{array}$ & $\phi_{\mathbf{0}}$ \\
\hline 0.166 & 11.1 & 0.0157 \\
0.255 & 6.80 & 0.0537 \\
\hline
\end{tabular}

Joumal of Colloid and Interface Science, Vol. 108, No. 2, December 1985 
of particles, $\phi_{0}$, was determined gravimetrically.

Bivalent electrolytes were used as flocculating agents. Bivalent electrolytes are preferred because the critical flocculation concentration is lower for these types of electrolytes than for monovalent ones, thus minimizing the increase in solvent viscosity and the time required for complete mixing of the diluted latex and flocculating agent. For rapid flocculating conditions, $\mathrm{BaCl}_{2}$ was used, whereas $\mathrm{MgSO}_{4}$ was used for slow flocculating conditions. A symmetric electrolyte was chosen for the slow flocculating experiments in order to conform with the electrostatic repulsion model given by [5].

$\zeta$ potential measurements were carried out on a LASER ZEE MODEL 500 with each monodisperse system at various $\mathrm{MgSO}_{4}$ concentrations. Since this $\zeta$ meter is calibrated for aqueous media at $20^{\circ} \mathrm{C}$, the $\zeta$ potentials were corrected for any differences between the standard and actual experimental conditions. The two particle systems gave the same results within experimental error, and the average values are shown in Fig. 1. The $\zeta$ potentials are usually substituted for $\Psi_{0}$ in the electric double layer repulsion model [5] even though there is no theoretical justification.

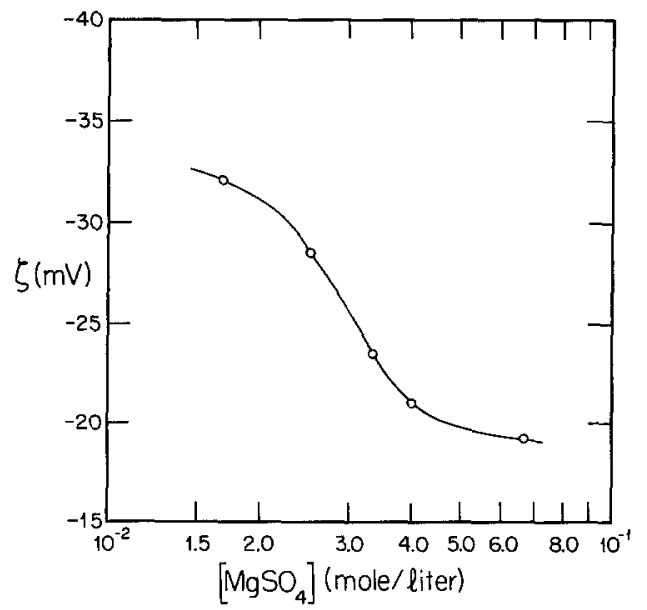

FIG. 1. Dependence of the average $\zeta$ potential on the $\mathrm{MgSO}_{4}$ concentration.

Journal of Colloid and Interface Science, Vol. 108, No. 2, December 1985

\subsection{Flocculation Rate Measurements}

The initial flocculation rates were measured using an extension of the stopped-flow turbidity technique developed by Lichtenbelt $e t$ al. (59) in which only flocculating monodisperse systems were considered. For an initially bidisperse system undergoing simultaneous flocculation and creaming, the rate constant $k_{12}$ for doublet formation between particles of radius $a_{1}$ and $a_{2}$ is related to the system turbidity $\tau$ through (60)

$$
\begin{aligned}
& k_{12}= \frac{1}{\tau_{0}}\left(\frac{d \tau}{d t}\right)_{0}\left\{\frac{1}{N_{02} F_{12}}+\frac{1}{N_{01} F_{21}}\right\} \\
&-\frac{k_{11} N_{01}}{N_{02}} \frac{F_{1}}{F_{12}}-\frac{k_{22} N_{02}}{N_{01}} \frac{F_{2}}{F_{21}} \\
&+\frac{K_{1}}{N_{02} F_{12}}+\frac{K_{2}}{N_{01} F_{21}},
\end{aligned}
$$

where the subscript 0 refers to the limit as the time $t \rightarrow 0$; $k_{i i}$ the homoflocculation rate constant between spheres of radius $a_{i} ; N_{0 i}$ the initial particle concentration for a sphere of radius $a_{i} ; F_{i}$ and $F_{i j}$ the optical factors given by

$$
F_{i}=\frac{C_{i i}}{2 C_{i}}-1
$$

and

$$
F_{i j}=\frac{C_{12}}{2 C_{i}}-\frac{C_{j}}{C_{i}}-1,
$$

with $C_{i}$ and $C_{i j}$ being the extinction cross sections of primary and doublet particles, respectively, and $K_{i}$ the creaming constant given by

$$
K_{i}=\frac{2 \Delta \rho \omega^{2} a_{i}^{2}}{9 \mu_{\mathrm{f}}},
$$

with $\omega$ being the angular velocity of a centrifuge rotor, $\Delta \rho$ the density difference between the particles and the suspending fluid, and $\mu_{\mathrm{f}}$ the viscosity of the suspending fluid.

Equation [6] indicates that the bidisperse rate constant $k_{12}$ can be determined directly from the measurable quantities $\tau_{0},(d \tau / d t)_{0}$, $N_{01}, N_{02}, K_{1}$, and $K_{2}$, and from the optical factors $F_{i}$ and $F_{i j}$ which can be computed from light scattering theory. However, before de- 
termining $k_{12}$, it is also necessary to determine the rate constants $k_{11}$ and $k_{22}$. These rate constants can be determined from either theoretical calculations using Brownian flocculation theory (5), or more rigorously, from initial rate experiments with each monodisperse sample under identical physical and chemical conditions. In this case $k_{11}$ can be determined from
[6] by simply setting $N_{02}=0$. The rate constant $k_{22}$ can be determined in an analogous manner by setting $N_{01}=0$.

The extinction cross sections can be computed from the Rayleigh-Gans-Debye (RGD) theory of light scattering $(61 \mathrm{a}, 62)$, and upon substituting these relationships into [7] gives the following expressions for the optical factors (60):

$$
\begin{gathered}
F_{i}=\frac{\int_{\xi=0}^{\pi} P_{i}^{s}(\xi)\left[\frac{\sin 2 v_{i}}{2 v_{i}}\right]\left(1+\cos ^{2} \xi\right) \sin \xi d \xi}{\int_{\xi=0}^{\pi} P_{i}^{s}(\xi)\left(1+\cos ^{2} \xi\right) \sin \xi d \xi}, \text { and } \\
F_{i j}=\frac{\left\{2.0 \int_{\xi=0}^{\pi}\left[P_{i}^{s}(\xi) P_{j}^{s}(\xi)\right]^{1 / 2}\left[\frac{\sin \left(1+\lambda_{i j}\right) v_{i}}{\left(1+\lambda_{i j}\right) v_{i}}\right]\left(1+\cos ^{2} \xi\right) \sin \xi d \xi\right\}}{\lambda_{i j}^{3} \int_{\xi=0}^{\pi} P_{i}^{s}(\xi)\left(1+\cos ^{2} \xi\right) \sin \xi d \xi},
\end{gathered}
$$

where

$$
\begin{gathered}
P_{i}^{s}(\xi)=9\left(\sin v_{i}-v_{i} \cos v_{i}\right)^{2} / v_{i}^{6}, \\
v_{i}=\frac{4 \pi n_{\mathrm{m}} a_{i}}{\lambda_{0}} \sin (\xi / 2),
\end{gathered}
$$

and

$$
\lambda_{i j}=a_{i} / a_{j}
$$

with $P_{i}^{s}(\xi)$ being the Rayleigh form factor for a sphere of radius $a_{i}, \xi$ the smallest angle between the incident and scattered beam, $n_{\mathrm{m}}$ the refractive index of the suspending medium, and $\lambda_{0}$ the wavelength of the incident beam in vacuo.

While the RGD theory is not strictly valid for particles with a diameter larger than approximately $0.26 \mu \mathrm{m}$, Egusa (63) has shown experimentally that the RGD theory can be used up to particle diameters of at least 0.765 $\mu \mathrm{m}$ since the optical factors in [6] depend only on the ratio of extinction cross sections. As a result, errors in $C_{1}$, etc., due to the application of the RGD theory tend to eliminate one another to a considerable extent in the estimation of the optical factors. It should also be pointed out that even though $C_{1}$ and $C_{2}$ can be computed from the exact Mie theory of light scat- tering $(61 \mathrm{~b})$, it is more convenient to use the RGD theory since the refractive indices of the particles need not be known.

\subsection{INITIAL RATE EXPERIMENTS}

\subsubsection{Brownian Flocculation Experiments}

Portions of the original latexes were first diluted with deionized water to a particle volume fraction ranging from approximately 2.0 $\times 10^{-5}$ to $10^{-4}$ prior to each set of experiments. One part of the flocculating agent was then rapidly mixed with two parts of the diluted latex to give the final particle and electrolyte concentrations. The change in optical density (which is directly proportional to the turbidity) was measured continuously using a Varian DMS 100 UV-Visible Spectrophotometer at a preselected wavelength. Depending on the particle system and the initial particle concentration, the time rate of change in optical density remained linear for at least $0.5 \mathrm{~min}$ and at most $4.5 \mathrm{~min}$. The initial slope $(d \tau / d t)_{0}$ was determined from the linear portion of the curve. It was impossible to obtain the turbidity 
of the flocculating suspension at time zero. Instead, a second mixture was diluted with deionized water to obtain the same initial particle concentration.

In order to assess the accuracy of the turbidity measurements obtained with the Varian spectrophotometer, the initial particle concentrations determined gravimetrically were compared to the concentrations obtained from turbidity measurements with $\tau_{0}=C_{i} N_{0 i}$ ( $i=1$ or 2 ). In all cases, the values of $N_{0 i}$ were approximately $7-10 \%$ lower with the turbidity measurements. This discrepancy is most likely due to optical imperfections of the spectrophotometer which usually result in an underestimation of the sample turbidity (64-68). However, it is assumed that any systematic error in $\tau$ is present to the same extent in $d \tau /$ $d t$, and therefore cancels itself in the quantity $\left(1 / \tau_{0}\right)(d \tau / d t)_{0}$. This assumption is acceptable because Beer's law was valid in the concentration range studied, indicating that any error in $\tau$, and therefore in $d \tau / d t$ also, would be a constant factor.

Each time a stopped-flow measurement was made, the sample temperature was recorded, and was used to calculate the fluid viscosity $\mu_{\mathrm{f}}$ from a correlation given in the "CRC Handbook of Chemistry and Physics" for water. Because of the low electrolyte concentrations used ( $\leqslant 0.07 M)$, the increase in solvent viscosity was less than $1 \%$ and, consequently, neglected in the data analysis.

\subsubsection{Enhanced Gravitational Flow Field Experiments}

Increased gravitational flow field experiments were carried out in a Fisher MicroCentrifuge Model 235A which has a maximum acceleration of $13,000 \times g$. Essentially the same experimental procedure was used in these sets of experiments as in the Brownian flocculation experiments. There were, however, some important differences. First of all, the change in optical density could not be measured continuously. Instead, parallel experiments were carried out with different mix- tures having the same initial conditions, but were centrifuged for varying lengths of time. In this way, a semicontinuous time rate of change in turbidity was obtained and, at the same time, sample disturbance was minimized since the centrifuge had to be started and stopped only once during each experiment. Multiple experiments were run for each time interval and the results were averaged in order to minimize the random error associated with measuring the initial volumes of latex and flocculating agent.

Secondly, during each experiment, the suspension would first undergo a period of only Brownian flocculation, the centrifuge would be started and the suspension would undergo gravity-enhanced/Brownian flocculation, and finally when the centrifuge was stopped to take a turbidity measurement, the suspension would again undergo only Brownian flocculation. The procedure used to correct the data for these periods of Brownian flocculation is analogous to the method used by Zeichner (69) and Feke (51) for their shear-induced flocculation experiments, and is given as follows. The period of time the suspension was in the Brownian flocculation mode, $t_{\mathrm{Br}}$, was recorded for each experiment (usually $30 \mathrm{~s}$ ). Assuming that the stability ratio remains constant from the one measured in the Brownian flocculation experiments, then the change in the measured turbidity, due to the periods of Brownian flocculation, is given through [6] as

$$
\frac{\Delta \tau}{\tau_{0}}=t_{\mathrm{Br}}\left[\frac{k_{12}+k_{11} \frac{N_{01}}{N_{02}} \frac{F_{1}}{F_{12}}+k_{22} \frac{N_{02}}{N_{01}} \frac{F_{2}}{F_{21}}}{\frac{1}{N_{02} F_{12}}+\frac{1}{N_{01} F_{21}}}\right]
$$

since $\omega=0$ under normal gravity conditions (note: $k_{12}$ is the bidisperse flocculation rate under normal gravity conditions). Therefore, the contribution to the total turbidity due to the periods of centrifuging is given by

$$
\tau_{\text {actual }}=\tau_{\text {measured }}-\Delta \tau\left(t_{\mathrm{Br}}\right) \text {. }
$$

Further details of the turbidity measurements and other experimental procedures are described by Melik (60). 


\section{RESULTS AND DISCUSSION}

\subsection{BROWNIAN FLOCCULATION EXPERIMENTS}

Rapid Brownian experiments were performed with each monodisperse system listed in Table I for a variety of initial particle concentrations and vacuum wavelengths (note: see reference 60 for complete results). A final $\mathrm{BaCl}_{2}$ concentration of $0.07 \mathrm{M}$ was used in these experiments, a value well above the critical flocculation concentration for polystyrene latexes (29). Brownian flocculation stability factors were computed from the initial flocculation rates through

$$
\begin{aligned}
W_{\mathrm{Br}} & =\frac{J_{\mathrm{Br}}^{0}}{k_{12}} \\
& =\frac{1}{k_{12}} \frac{2 k T}{3 \mu_{\mathrm{f}}}\left(\frac{1}{a_{1}}+\frac{1}{a_{2}}\right)\left(a_{1}+a_{2}\right),
\end{aligned}
$$

where $J_{\mathrm{Br}}^{0}$ is the Smoluchowski Brownian collision rate, $k$ the Boltzmann constant, and $T$ the absolute temperature. The average stability factors are listed in Table II. Since the 95\% confidence intervals for each particle system overlap, they have essentially the same initial flocculation rate under rapid conditions with an overall stability factor of $W_{\mathrm{Br}}=2.10 \pm 0.02$. The Hamaker constant can be estimated by comparing the measured stability factor with those predicted from Brownian flocculation theory (5), namely

$$
W_{\mathrm{Br}}=2.0 \int_{R=2}^{\infty} \frac{e^{V_{\mathrm{int} / k T}}}{G(R, \lambda) R^{2}} d R,
$$

\section{TABLE II}

Rapid Brownian Flocculation Results with the Monodisperse Systems

\begin{tabular}{cccc}
\hline & & \multicolumn{2}{c}{$A \times 10^{14}(\mathrm{ergs})$} \\
\cline { 3 - 4 }$a(\mu \mathrm{m})$ & $W_{\text {Br }}$ & $\nu=\infty$ & $\lambda_{\mathrm{L}}=10^{-3} \mathrm{~cm}$ \\
\hline 0.166 & $2.08 \pm 0.03$ & $2.00 \pm 0.29$ & $2.82 \pm 0.48$ \\
0.255 & $2.11 \pm 0.04$ & $1.76 \pm 0.33$ & $2.99 \pm 0.70$ \\
Overall & $2.10 \pm 0.02$ & $1.83 \pm 0.19$ & $2.87 \pm 0.36$ \\
\hline
\end{tabular}

where $G(R, \lambda)$ is a hydrodynamic correction function (60). Under rapid flocculating conditions $V_{\mathrm{R}} \approx 0$, and $V_{\text {int }}$ is dominated by $V_{\mathrm{A}}$ in which the Hamaker constant is the only unknown. The resulting Hamaker constants determined by trial and error for both an unretarded and retarded, with $\lambda_{\mathrm{L}}=10^{-5} \mathrm{~cm}$, attractive potential are listed in Table II.

These values of the Hamaker constant fall within the range of Hamaker constants for polystyrene latexes reviewed by Zeichner and Schowalter (18): from $1.7 \times 10^{-14}$ to 1.7 $\times 10^{-13}$ erg. Althơugh the present values are closer to the lower end than the average, confidence can be given to the present value since Zeichner and Schowalter (18) report a Hamaker constant of $A=2.0 \pm 0.6 \times 10^{-14} \mathrm{erg}$ from both their shear-induced flocculation and Brownian flocculation experiments, as opposed to the larger values which were determined solely from rapid Brownian flocculation experiments.

The overall stability factor for a bidisperse mixture with $N_{01} / N_{02} \simeq 1.06$ was determined to be $W_{\mathrm{Br}}=2.02 \pm 0.12$. (Note: in a separate analysis (70), it was shown that this particle concentration ratio approximately maximizes the net particle loss rate of gravity-induced flocculation as compared to particle creaming.) This decrease in the stability factor with an increase in the particle-size spread has also been observed by Matthews and Rhodes (32), and can be predicted from Brownian flocculation theory by means of [14]. Using the overall Hamaker constants from Table II, a stability factor of $W_{\mathrm{Br}}=2.05 \pm 0.03$ is obtained for an unretarded potential, and a value of $W_{\mathrm{Br}}=2.04 \pm 0.04$ is obtained for a retarded potential.

The slow Brownian flocculation results are shown in Fig. 2 for both monodisperse systems, where the critical flocculation concentration is approximately $0.04 \mathrm{M} \mathrm{MgSO}_{4}$ for $a$ $=0.166 \mu \mathrm{m}$ and approximately $0.049 M$ for $a=0.255 \mu \mathrm{m}$. The increase in magnitude of the Brownian flocculation stability factor with decreasing electrolyte concentration indicates the presence of electrostatic stabilization and 
justifies the use of the DLVO theory to describe the colloidal behavior of polystyrene latexes. In addition, the increase in the critical flocculation concentration with increasing particle size is also in accordance with the particle size dependence predicted by DLVO theory.

Just as the rapid Brownian flocculation experiments can be used to characterize the system Hamaker constant, slow Brownian experiments can be used to estimate the surface potential $\Psi_{0}$. When electrostatic stabilization is significant, the stability factor $W_{\mathrm{Br}}$ of a monodisperse system with radius $a$ should vary with the electrolyte concentration $C_{\mathrm{E}}$ of the solution as follows (25):

where

$$
\ln W_{\mathrm{Br}}=k_{1} \ln C_{\mathrm{E}}+k_{2} \text {, }
$$

$$
k_{1}=\frac{-1.472 \epsilon k T}{e^{2}} \frac{a \gamma^{2}}{z^{2}}
$$

and

$$
\gamma=\frac{\exp \left[z e \Psi_{0} / 2 k T\right]-1}{\exp \left[z e \Psi_{0} / 2 k T\right]+1}
$$

with $e$ being the electron charge and $z$ the valence of the symmetric electrolyte in solution. From a linear regression of the stability factors in Fig. 2 which follow the relationship [15], a value for the surface potential of $\Psi_{0} \simeq 10.3$ $\mathrm{mV}$ (disregarding the sign) is obtained for $a=0.166 \mu \mathrm{m}$ and a value of $\Psi_{0} \simeq 6.16 \mathrm{mV}$ is obtained for $a=0.255 \mu \mathrm{m}$. These surface potentials are lower than the $\zeta$ potentials shown in Fig. 1. This discrepancy is not surprising, especially considering the assumptions invoked in arriving at [15] from [14].

In order to more rigorously compute the surface potential from the slope concatenate, the exact relationship between $\ln W_{\mathrm{Br}}$ and $\ln$ $C_{\mathrm{E}}$ was used, namely (60)

$$
\frac{d \ln W_{\mathrm{Br}}}{d \ln C_{\mathrm{E}}}=\frac{-\kappa N_{\mathrm{A}} N_{\mathrm{R}} \int_{R=2}^{\infty} \frac{(R-2) \exp [-\kappa(R-2)]}{\{1+\exp [-\kappa(R-2)]\}} \frac{e^{V_{\mathrm{in} / k T}}}{G(R, \lambda) R^{2}} d R}{2.0 \int_{R=2}^{\infty} \frac{e^{V_{\mathrm{in}} / k T}}{G(R, \lambda) R^{2}} d R}
$$

where

$$
N_{\mathrm{A}}=\frac{2 A}{k T} \frac{\lambda}{(1+\lambda)^{2}},
$$

and

$$
N_{\mathrm{R}}=\frac{\epsilon \Psi_{0}^{2}\left(a_{1}+a_{2}\right)}{2 A} .
$$

Unlike the constant slope concatenate predicted by [15], numerical integration of [16] shows a definite dependence of this slope on the electrolyte concentration $C_{\mathrm{E}}\left(C_{\mathrm{E}} \propto \kappa^{2}\right)$ (71). Consequently, in order to estimate the surface potential $\Psi_{0}$, a regression analysis was performed on the experimental data shown in Fig. 2 to determine the value of $N_{\mathrm{R}}$ which resulted in the minimum value of

$$
\sum_{j=1}^{n}\left[\left(\frac{d \ln W_{\mathrm{Br}}}{d \ln C_{\mathrm{E}}}\right)_{\text {theory }}-\left(\frac{d \ln W_{\mathrm{Br}}}{d \ln C_{\mathrm{E}}}\right)_{\text {exp }}\right]^{2},
$$

where $n$ is the number of regression points. The results of this analysis are summarized in Table III for both an unretarded and retarded, with $\lambda_{\mathrm{L}}=10^{-5} \mathrm{~cm}$, attractive potential. The magnitude of these surface potentials are larger than those determined from [15], but they are still lower than the measured $\zeta$ potentials. As shown in Figs. 3 and 4, the values of the stability factors computed with these surface potentials, and the overall Hamaker constants listed in Table II, moderately agree for the particle system with $a=0.166 \mu \mathrm{m}$, but are completely erroneous for the particle system with $a=0.255 \mu \mathrm{m}$.

Because of this discrepancy between the experimentally measured and theoretically predicted stability factors, a regression analysis based on the actual value of the stability factor was also carried out. In this case, a search was 


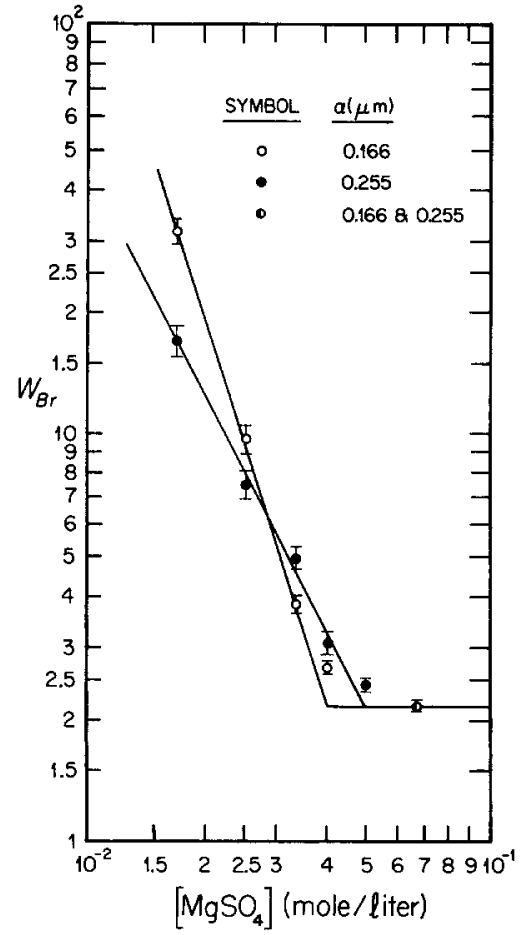

Fig. 2. Effect of electrolyte concentration on the Brownian flocculation stability factor. (Note: both particle sizes give the same flocculation rate for $0.0667 \mathrm{MMgSO}_{4}$.)

performed to determine the value of $N_{\mathrm{R}}$ which resulted in the minimum value of

$$
\sum_{j=1}^{n}\left[\frac{\left(\ln W_{\mathrm{Br}}\right)_{\text {theory }}-\left(\ln W_{\mathrm{Br}}\right)_{\mathrm{exp}}}{\left(\ln W_{\mathrm{Br}}\right)_{\exp }}\right]^{2} .
$$

By choosing the logarithm of the stability factor as the regression variable instead of $W_{\mathrm{Br}}$, the more precisely measured fast flocculation rates (smaller stability factors) are weighted

\section{TABLE III}

Values of $\Psi_{0}$ by Regression Analysis of the Slow Brownian Flocculation Results

\begin{tabular}{|c|c|c|c|c|}
\hline \multirow{3}{*}{$\begin{array}{c}a \\
(\mu \mathrm{m})\end{array}$} & \multicolumn{4}{|c|}{ Regression variable } \\
\hline & \multicolumn{2}{|c|}{$d \ln W_{\mathrm{Br}} / d \ln C_{\mathrm{E}}$} & \multicolumn{2}{|c|}{$\ln W_{\mathrm{Br}}$} \\
\hline & $\nu=\infty$ & $\lambda_{\mathrm{L}}=10^{-5} \mathrm{~cm}$ & $\nu=\infty$ & $\lambda_{\mathrm{L}}=10^{-5} \mathrm{~cm}$ \\
\hline 0.166 & $13.8 \mathrm{mV}$ & $16.2 \mathrm{mV}$ & $13.2 \mathrm{mV}$ & $16.3 \mathrm{mV}$ \\
\hline 0.255 & $11.3 \mathrm{mV}$ & $12.7 \mathrm{mV}$ & $12.2 \mathrm{mV}$ & $14.1 \mathrm{mV}$ \\
\hline
\end{tabular}

more than the less precise slower rates. The results of this analysis are also summarized in Table III. It is evident from this table that both regression analyses give similar values for $\Psi_{0}$. However, as can be seen from Figs. 3 and 4, the regression variable $\ln W_{\mathrm{Br}}$ yields a value of $\Psi_{0}$ which results in a better agreement between theory and experiment.

In order to further investigate the potential usefulness of this type of analysis, the measured bidisperse stability factors were compared with the predicted ones computed using the surface potentials given in Table III, with ln $W_{\mathrm{Br}}$ as the regression variable, and the $\zeta$ potentials shown in Fig. 1. Since the surface potentials of each monodisperse system are slightly different, and since the chosen electrostatic repulsion model [5] assumes each particle has the same surface potential, an average potential given by

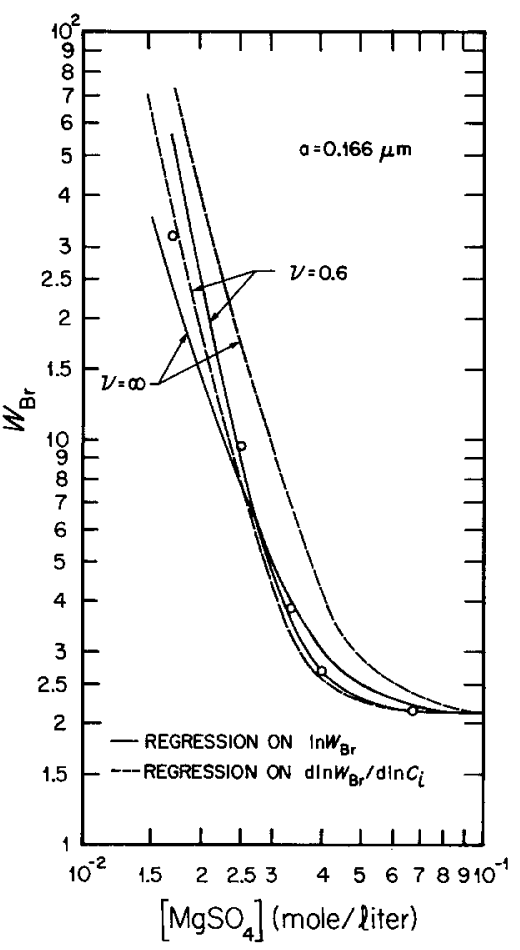

FIG. 3. Comparison of experimental and theoretical Brownian flocculation stability factors computed with the surface potentials determined from the regression analysis for $a=0.166 \mu \mathrm{m}$. 


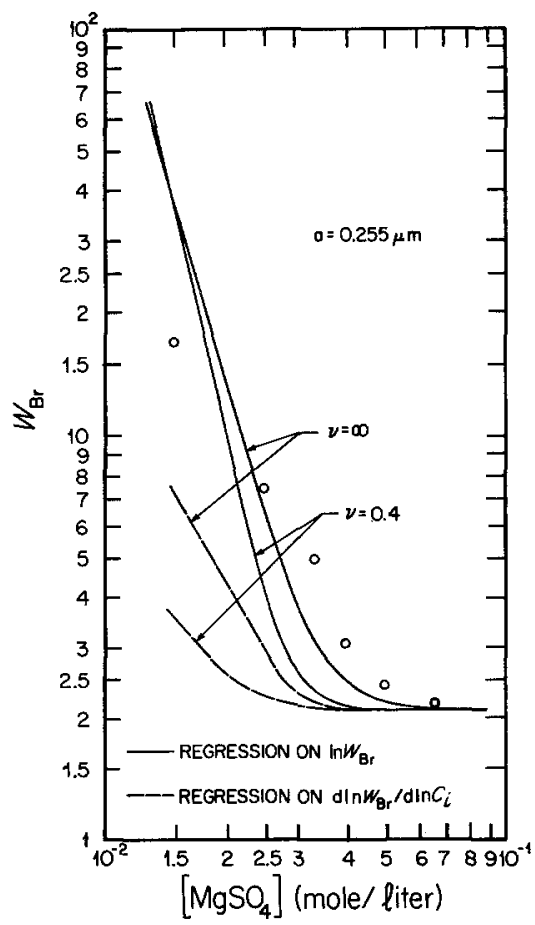

FIG. 4. Comparison of experimental and theoretical Brownian flocculation stability factors computed with the surface potentials determined from the regression analysis for $a=0.255 \mu \mathrm{m}$.

$$
\left\langle\Psi_{0}^{2}\right\rangle=\frac{1}{2}\left(\Psi_{01}^{2}+\Psi_{02}^{2}\right)
$$

was used. A comparison of the repulsive forces computed from [5] using $\left\langle\Psi_{0}^{2}\right\rangle$, with the more general model which assumes the potentials are different (31), shows less than a $1 \%$ difference. The results of the stability factor computations are shown in Fig. 5. It is evident from this figure that the surface potentials determined from the regression analysis give much better stability predictions than the $\zeta$ potentials.

\subsection{GRAVITY-ENHANCED FLOCCULATION EXPERIMENTS}

Initial flocculation rates were measured under conditions of an enhanced gravitational flow field on a bidisperse mixture with $N_{01} /$ $N_{02} \simeq 1.06$. As shown elsewhere (70), this particle concentration ratio approximately maximizes the net particle loss rate of gravity-in-

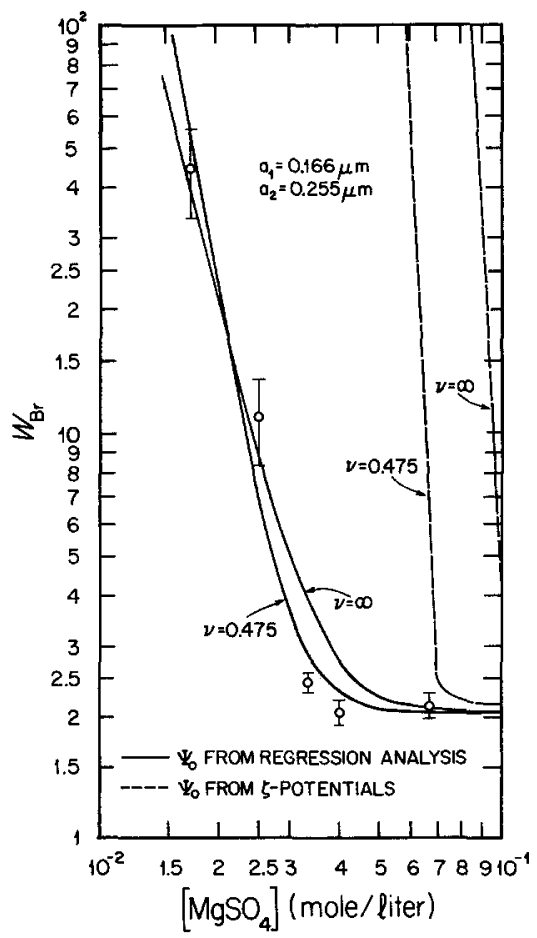

FIG. 5. Comparison of experimental and theoretical Brownian flocculation stability factors computed with the surface potentials from the regression analysis and with the $\zeta$ potentials for the bidisperse mixture.

duced flocculation as compared to particle creaming. Only rapid flocculating conditions were successfully investigated. Problems with slow flocculating conditions are discussed elsewhere (60).

The rapid gravity-enhanced flocculation experiments are summarized in Table IV, where the capture efficiency $\alpha=k_{12} / J_{\mathrm{Br}}^{0}$ with $J_{\mathrm{Br}}^{0}$ being given by [13]. A final $\mathrm{BaCl}_{2}$ concen-

TABLE IV

Rapid Gravity-Enhanced Flocculation Results

\begin{tabular}{rccc}
\hline $\begin{array}{c}g \\
\text { force }\end{array}$ & $\begin{array}{c}\langle T\rangle \\
\left({ }^{\circ} \mathrm{C}\right)\end{array}$ & $\begin{array}{c}k_{12} \\
\left(\mathrm{~cm}^{3} / \mathrm{min}\right)\end{array}$ & $\alpha=k_{12} / J_{\text {brt }}^{0}$ \\
\hline 1.0 & 26.4 & $3.97 \pm 0.24 \times 10^{-10}$ & $0.495 \pm 0.030$ \\
36.0 & 24.9 & $3.88 \pm 0.24 \times 10^{-10}$ & $0.502 \pm 0.030$ \\
170.0 & 22.9 & $4.33 \pm 0.25 \times 10^{-10}$ & $0.591 \pm 0.034$ \\
758.0 & 27.5 & $1.44 \pm 0.21 \times 10^{-9}$ & $1.74 \pm 0.25$ \\
$2,139.0$ & 27.0 & $1.76 \pm 0.58 \times 10^{-9}$ & $2.16 \pm 0.71$ \\
$6,266.0$ & 27.9 & $3.16 \pm 4.53 \times 10^{-10}$ & $0.379 \pm 0.543$ \\
$13,000.0$ & 27.9 & $-1.12 \pm 1.12 \times 10^{-9}$ & $-1.34 \pm 1.34$ \\
\hline
\end{tabular}


tration of $0.0533 M$ was used in this series of experiments, and the change in turbidity was recorded at a vacuum wavelength of $\lambda_{0}=546$ $\mathrm{nm}$. The observation times for the lower $g$ forces $(\leqslant 170)$ were on the order of $30 \mathrm{~s}$, whereas observation times for the larger $g$ forces were on the order of 5-10 s. For longer time periods, the effects of creaming would start dominating the flocculation process, and the turbidity would decrease instead of increase with time. In fact, for the highest $g$ force listed in Table IV, creaming completely dominated any flocculation, thereby giving a negative flocculation rate. This result indicates that for the higher gravitational forces, the restriction to initial times inherent in [6] was being violated even with the short observation times obtained in the present experiments. Either shorter experimental times are required (maybe down to $1 / 10$ of a second or less), or a more rigorous analysis, which would then require numerical computations $(60)$, is necessary to determine the rate constant $k_{12}$ at the higher $g$ forces.

In spite of this shortcoming, it is evident from Table IV that the flocculation rate increases as the gravitational force is increased. For small gravitational forces, Melik and Fogler (11) found that the capture efficiency $\alpha$ is given by

$$
\alpha=\frac{1}{W_{\mathrm{Br}}}\left(1+\frac{\mathrm{Gr}}{W_{\mathrm{Br}}}\right)+O\left(\mathrm{Gr}^{2} \ln \mathrm{Gr}\right),
$$

where the gravity number is defined by

$$
\mathrm{Gr}=\frac{2 \pi g \Delta \rho a_{2}^{4}}{3 k T} \lambda\left(1-\lambda^{2}\right)
$$

and represents the relative importance of gravitational forces and Brownian motion, with $g$ being the local acceleration of gravity. Equation [22] was derived by means of a singular perturbation analysis of the governing convective-diffusion equation (11). As shown in Fig. 6 for the lower gravitational forces $(\mathrm{Gr}$ $<0.1$ ), the perturbation solution given by [22] correctly predicts the minor effect of gravity on the bidisperse flocculation rate.

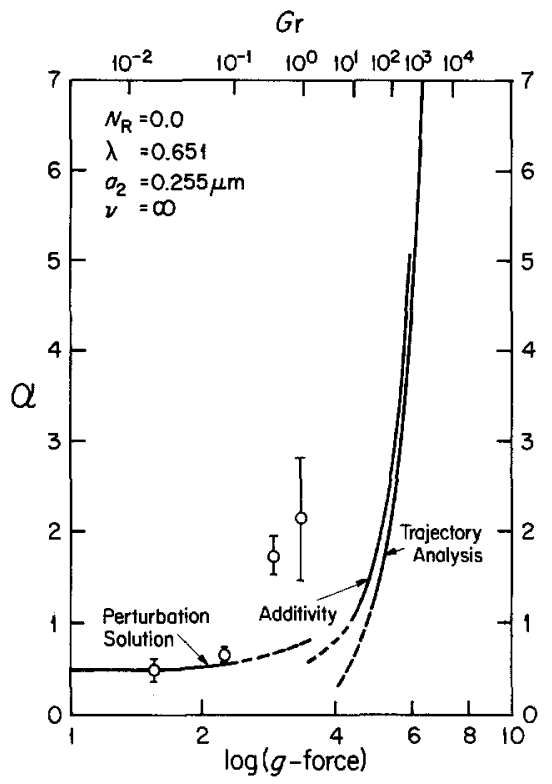

FIG. 6. Comparison of the experimentally observed and the theoretically predicted rapid flocculation rates for an enhanced gravitational flow field.

For large gravitational forces $(\mathrm{Gr} \gg 1)$, the capture efficiency can be determined by solving the governing trajectory equations. Melik and Fogler (10) and Davis (12) show that in the absence of Brownian motion, the trajectory equations take the following form:

$$
\begin{gathered}
\frac{d R}{d t}=-A(R, \lambda) \cos \theta-\frac{G(R, \lambda)}{\mathrm{Gr}} \frac{1}{k T} \frac{\partial V_{\mathrm{int}}}{\partial R}, \\
\frac{d \theta}{d t}=\frac{B(R, \lambda) \sin \theta}{R},
\end{gathered}
$$

and

$$
\frac{d \phi}{d t}=0
$$

where $\theta$ and $\phi$ are spherical polar coordinates with gravity acting in the $\theta=\pi$ direction, $A(R$, $\lambda), B(R, \lambda)$, and $G(R, \lambda)$ the hydrodynamic correction functions (10), and $t$ the time. By integrating [24], the limiting trajectory for particle capture can be located and used to compute the capture efficiency (10). This limiting trajectory corresponds to those trajectories furthest from the reference sphere which still lead to particle capture and are charac- 
terized by the upstream coordinates $R=\infty$ and $\theta=\theta_{\lim }$. For angular positions $\theta<\theta_{\mathrm{lim}}$ when $R=\infty$, particle capture occurs. For angular positions $\theta>\theta_{\mathrm{lim}}$, the larger sphere will approach the smaller reference sphere, but as a result of the net repulsion between the particles at small interparticle distances (due to hydrodynamic and surface interactions), the particles will move past one another and separate after an encounter.

Due to the experimental limitations discussed above for the higher $g$ forces, a quantitative verification of this trajectory analysis was not possible. For the gravitational forces at which rate data were obtained, the qualitative direction of the data is in agreement with the trajectory analysis; both indicate an increase in the flocculation rate with an increase in the gravitational force (see Fig. 6). In addition, the experimental results indicate a high degree of coupling between Brownian motion and differential creaming. When the simplistic assumption of summing the individual gravity-induced and Brownian flocculation rates is invoked, the observed flocculation rates are still a factor of approximately 3.0 larger than predicted. This coupling phenomenon can be completely resolved only by solving the model for flocculation in quiescent media given by Melik and Fogler (11) for intermediate gravitational forces (i.e., $0.1<\mathrm{Gr}<10.0$ ).

\subsection{APPLICABILITY OF THE DLVO THEORY}

The inadequacy of the classical DLVO theory that becomes apparent when using $\zeta$ potentials in place of $\Psi_{0}$ in [5], as illustrated in Fig. 5, probably results from either an underestimation of the net attraction between particles, or from an overestimation of the repulsive force. Both of these possible errors are discussed in light of the present results.

First of all, we consider the underestimation of the attractive potential by comparing our results with the rigorous Lifshitz theory. The analysis of Parsegian and Ninham (23) using Lifshitz theory shows that electromagnetic dispersion interactions include contributions from the infrared and microwave frequencies in addition to the ultraviolet contribution. This latter contribution can be approximately identified with the classical Hamaker dispersion interaction. In the absence of electrolyte and retardation effects, Parsegian and Weiss (72) computed a Hamaker constant of $A=1.3$ $\times 10^{-13}$ erg for polystyrene particles dispersed in water, approximately an order of magnitude larger than that determined from our rapid Brownian experiments.

When the effects of retardation and high electrolyte concentrations are considered, the Hamaker constant decreases with increasing interparticle separation. The calculations of Parsegian and Ninham (23) show that the presence of electrolytes sharply reduces the microwave contribution for interparticle separations greater than the double layer thickness $1 / \kappa$; whereas, the infrared and ultraviolet contributions remain essentially unaffected. Under rapid flocculating conditions the double layer is totally collapsed and, therefore, the microwave contribution is negligible. Consequently, since the microwave portion contributes approximately $25 \%$ to the total dispersion attraction (46), the total Hamaker constant for polystyrene particles in water should be no larger than approximately $9.8 \times 10^{-14} \mathrm{erg}$ under high electrolyte concentrations.

Retardation can also significantly affect the magnitude of the Hamaker constant. The recent Lifshitz calculations of Pailthorpe and Russel (48) show that in the presence of high electrolyte concentrations, the Hamaker constant for polystyrene spheres in water is reduced by approximately $70 \%$ for $a=0.1 \mu \mathrm{m}$ and by approximately $87 \%$ for $a=0.25 \mu \mathrm{m}$ in the range $2.001<R<2.5$. Consequently, the expected value of the Hamaker constant for our particle system should lie somewhere in the range from $1.7 \times 10^{-14}$ to $9.8 \times 10^{-14}$ erg. Therefore, the effects of retardation and electrolyte concentration help explain the discrepancy between our rapid Brownian flocculation results and the Lifshitz theory calculations for polystyrene in water. This result implies that the attractive potential can be ad- 
equately computed from [2] or [3] for systems other than polystyrene in water provided that the Hamaker constant is either determined experimentally or calculated from Lifshitz theory.

We now address the possibility that the repulsive force is overpredicted. If we assume that the repulsive potential computed from [5] is correct when using $\zeta$ potentials in place of $\Psi_{0}$, then for the results shown in Fig. 5 agreement between theory and experiment can be accomplished only for a Hamaker constant that increases with decreasing salt concentration. Using the measured $\zeta$ potentials, it is found that the Hamaker constant must increase from approximately $3.7 \times 10^{-14} \mathrm{erg}$ at $0.0667 \mathrm{M} \mathrm{MgSO}_{4}$ to a value greater than approximately $1.9 \times 10^{-13} \mathrm{erg}$ for concentrations less than $0.0167 \mathrm{MMgSO}_{4}$ with $a=0.166 \mu \mathrm{m}$ and $\nu=\infty$. Similar ranges for the Hamaker constant are found for $a=0.255 \mu \mathrm{m}$ and $\nu=\infty$, and for a retarded potential with both particle systems. In view of the Lifshitz calculations, which predict a difference of at most $3.0 \times 10^{-14}$ erg due to electrolyte concentration (46), it seems more plausible that the double layer repulsive forces estimated with the Hogg, Healy, and Fuerstenau (HHF) model, Eq. [5], are too large.

The results in Fig. 5 also show another important discrepancy between the experimentally measured stability factors and those predicted from the HHF model using $\zeta$ potentials: the slopes concatenate do not agree. Duckworth and Lips (73) suggest that this discrepancy could be due to the adsorption of ions in the Stern layer. In this case, the interparticle distance $R$ in the HHF model is replaced with $R-\delta$, where $\delta$ is the Stern layer thickness. However, while realistic values of the thickness $\delta$ were obtained (on the order of $10-50 \mathrm{~nm}$, the size range of hydrated ions expected with the Stern layer concept), this approach could not completely account for the insensitivity of the slopes concatenate to changes in the particle size (73).

Prieve and Lin (74) suggest that the discrepancy between the theoretical (using $\zeta$ for
$\left.\Psi_{0}\right)$ and the experimental slopes may be due to a distribution in the surface potential of the polystyrene latexes. Their calculations show that a 5\% standard deviation in the surface potential can halve the value of concatenate for colloidal systems with an average radius of $0.1 \mu \mathrm{m}$ and a surface potential between 30 and $200 \mathrm{mV}$. For surface potentials less than 25 $\mathrm{mV}$, there is less than a $10 \%$ deviation in the slope. These results indicate that a distribution in the surface potential may be contributing to the discrepancy in the slopes illustrated in Fig. 5. However, considering that the slopes calculated using the $\zeta$ potentials are a factor of approximately 30 larger than the experimentally measured one, either there is a very broad surface potential distribution, which does not seem very plausible for our system, or as stated above, the HHF model, Eq. [5], simply overestimates the magnitude of the repulsive force.

Barouch and Matijevic $(55,78)$ also point out that the calculated repulsion energies from [5] often exceed the values suggested by experiments. It was suspected that treating the system as spherically symmetric (in which case the one-dimensional linear Poisson-Boltzmann equation can be used) was one of the main reasons for these discrepancies. To overcome this difficulty, the repulsion potentials were computed from the two-dimensional nonlinear Poisson-Boltzmann equation (55, 56). Unfortunately, this analysis involves very cumbersome numerical computations. However, numerical calculations of the electrostatic energies show that the values computed from [5] only overestimate the more accurately computed potentials by a constant factor for $R>2.001$ (55). This result indicates that the regression analysis proposed in this paper to estimate the effective surface potential with the HHF model determines a value of $\Psi_{0}^{2}$ which is lower by this same constant factor. Therefore, using this effective value of $\Psi_{0}^{2}$ results in a computed repulsion energy from [5] which is in fair agreement with the more rigorous computations of Barouch and Matijevic (55, $56,78)$. This result implies that the repulsive 
potential for polystyrene particles in water can be adequately estimated from the HHF model as long as the effective surface potential is determined experimentally and the measured $\zeta$ potentials are not used in place of $\Psi_{0}$.

While we have shown that the HHF model of electrostatic repulsion is appropriate for polystyrene particles in water, the general applicability of a constant surface potential model remains in question. There is much evidence in the literature to suggest that, in general, particle/particle encounters proceed on a time scale somewhere between the characteristic times associated with constant surface potential and constant surface charge conditions $(75,76)$. Unfortunately, there has been no complete analysis of the dynamic effects associated with overlapping electric double layers.

Weaver and Feke (77) have recently analyzed the time variation of the electrostatic repulsion force between colloidal plates and spheres at a fixed interparticle distance, thereby giving some insight into the importance of aging in colloidal flocs. The relaxation processes governing the redistribution of charges on the particle surface and the interchange of charge between the particle surface and the diffused part of the double layer were considered in the analysis. For flat plates, the electrostatic repulsion force decays exponentially from its initial value, which corresponds to the limiting case of constant charge interaction, to the equilibrium value corresponding to the constant potential case.

The double layer interaction between two equally sized spheres at a fixed interparticle separation is not so clearly defined. For the case when relaxation occurs primarily through the interchange between the particle surface and the diffuse part of the double layer, the repulsion force decays exponentially until at equilibrium the surface potential becomes constant. For the case when relaxation occurs preferentially through the redistribution of charges on the particle surfaces, the electrostatic repulsion force also decays exponentially, but the magnitude of the decay is smaller than in the previous case. When both relaxa-

Journal of Colloid and Interface Science, Vol. 108, No. 2, December 1985 tion processes occur simultaneously, the steady-state surface potential only attains its equilibrium value when surface diffusion of the charges does not occur. In all other cases, the average surface charge continually decreases during the course of a particle/particle encounter, indicating that the long-term electrostatic repulsion is greater than that predicted by constant potential theory.

These results indicate that the application of either a constant surface charge model or a constant surface potential model only give extreme values of the actual repulsion force. The lower bound is given by the constant potential case, whereas the upper bound is given by the constant charge case. It is clear, however, that the dynamics of the particle/particle interaction process are important in predicting both the magnitude and the decay rate of the repulsion force. Consequently, incorporation of double layer relaxation effects into interaction models will give a more realistic picture of particle/particle encounters. Unfortunately, the complexity of this incorporation is beyond the scope of this paper and will remain an important research problem for some time.

Overall though, in spite of the shortcomings associated with the classical interparticle potential model, this older approach continues to be widely used, mainly because of its greater convenience and the fact that, for many practical systems, the stability predictions are fairly good. The use of rapid Brownian experiments provides a simple method for determining a single-valued Hamaker constant that remains valid even when the repulsive force is significant because particle/particle interactions occur at approximately the same interparticle separation under both rapid and slow flocculating conditions. Consequently, the spatial dependence of the Hamaker constant is minimized. In addition, the regression analysis approach with the slow Brownian experiments provides a fairly simple method of obtaining an effective value of $\Psi_{0}$. This value of $\Psi_{0}$ best characterizes a given colloidal system in the sense of being able to satisfactorily predict colloidal stability under various physical and chemical conditions using the chosen inter- 
particle potential model. The proof of these arguments is borne out in Fig. 5.

\section{SUMMARY}

One set of initial flocculation rate experiments was carried out with two monodisperse suspensions of differing sized polystyrene spheres under normal gravity conditions. By comparing the measured stability factors under rapid flocculating conditions to the stability factors predicted by Brownian flocculation theory, a Hamaker constant of $A=2.87$ $\pm 0.19 \times 10^{-14} \mathrm{erg}$ was found for a retarded attractive potential. This value agrees fairly well with the Lifshitz theory which predicts that the Hamaker constant should decrease from a value of at most $9.8 \times 10^{-14} \mathrm{erg}$ at interparticle distances $R=2.001$ to a value of $1.7 \times 10^{-14}$ erg for $R=2.5$. However, considering that our value of the Hamaker constant was lower than most values previously reported in the literature, the only safe conclusion is that the experiments do not disprove the theory.

Using slow Brownian flocculation experiments, we estimated a characteristic potential by means of a regression analysis. This estimated potential has been shown to give good stability predictions with the Hogg, Healy, and Fuerstenau electrostatic repulsion model. Using the measured $\zeta$ potentials in place of the characteristic potential in their model greatly overestimates the stability of a colloidal system under low electrolyte concentrations.

In another set of experiments, we measured the bidisperse flocculation rate under conditions of an enhanced gravitational flow field, achieved by means of a centrifuge. As the gravitational force is increased, the effect of differential creaming becomes significant and the flocculation rate increases dramatically with increasing gravitational forces. For the lower gravitational forces, the perturbation analysis presented by Melik and Fogler (10) correctly predicts the effect of gravity on the bidisperse flocculation rate. Due to the experimental problem of particle creaming at the higher gravitational forces, quantitative verification of the trajectory analysis presented by
Melik and Fogler (11) and Davis (12) was not possible. However, for the gravitational forces at which rate data were obtained, the qualitative direction of the rate data and the trajectory analysis agree. In addition, our experimental results indicate a high degree of coupling between Brownian motion and gravitational forces. The intricacies of this association can only be studied by solving the complete convective-diffusion equation for quiescent media given by Melik and Fogler(11).

\section{REFERENCES}

1. Dickinson, E., J. Colloid Interface Sci. 73, 578 (1980).

2. Reed, C. C., and Anderson, J. L., AIChE J. 26, 816 (1980).

3. Batchelor, G. K., and Wen, C.-S., J. Fluid Mech. 124, 495 (1982).

4. Derjaguin, B. V., and Muller, V. M., Dokl. Akad. Nauk. SSSR (Engl. Transl.) 176, 738 (1967).

5. Spielman, L. A., J. Colloid Interface Sci. 33, 562 (1970).

6. Roebersen, G. J., and Wiersema, P. H., J. Colloid Interface Sci. 49, 98 (1974).

7. Kim, J. S., and Rajagopalan, R., Colloid Surf. 4, 17 (1982).

8. Feke, D. L., and Prabhu, N., "Kinetics of Coupled Primary- and Secondary-Minimum Coagulation in Colloidal Dispersions," Presented at the AIChE Annual Meeting, Los Angeles, Ca. (Nov. 14-19, 1982).

9. Valioulis, I. A., and List, E. J., Adv. Colloid Interface Sci. 20, 1 (1984).

10. Melik, D. H., and Fogler, H. S., J. Colloid Interface Sci. 101, 72 (1984).

11. Melik, D. H., and Fogler, H. S., J. Colloid Interface Sci. 101, 85 (1984).

12. Davis, R. H., J. Fluid Mech. 145, 179 (1984).

13. Wen, C.-S., and Batchelor, G. K., Sci. Sin. (series A), 28, 172 (1985).

14. Curtis, A. S. G., and Hocking, L. M., Trans. Faraday Soc. 66, 1381 (1970).

15. Van de Ven, T. G. M., and Mason, S. G., J. Colloid Interface Sci. 57, 505 (1976).

16. Van de Ven, T. G. M., and Mason, S. G., Colloid Polym. Sci. 255, 468 (1977).

17. Zeichner, G. R., and Schowalter, W. R., AIChE J. 23, 243 (1977).

18. Zeichner, G. R., and Schowalter, W. R., J. Colloid Interface Sci. 71, 237 (1979).

19. Adler, P. M., J. Colloid Interface Sci. 83, 106 (1981).

20. Feke, D. L., and Schowalter, W. R., J. Fluid Mech. 133, 17 (1983).

21. Derjaguin, B., and Landau, L., Acta. Physiochim. 14, 633 (1941).

22. Verwey, E. J. W., and Overbeek, J., Th. G., "Theory

Journal of Colloid and Interface Science, Vol. 108, No. 2, December 1985 
of the Stability of Lyophobic Colloids." Elsevier, Amsterdam, 1948.

23. Parsegian, V. A., and Ninham, B. W., J. Colloid Interface Sci. 37, 332 (1971).

24. Webb, J. T., Bhatnagar, P. D., and Williams, D. G., J. Colloid Interface Sci. 49, 346 (1974).

25. Reerink, H., and Overbeek, J. Th. G., Disc. Faraday Soc. 18, 74 (1954).

26. Schenkel, J. H., and Kitchener, J. A., Trans. Faraday Soc. 56, 161 (1960).

27. Ottewill, R. H., and Shaw, J. N., Disc. Faraday Soc. 42, 154 (1966).

28. Lips, A., and Willis, E., J. Chem. Soc. Faraday Trans. I 69, 1226 (1973).

29. Lichtenbelt, J. W. TH., Pathmamanoharan, C., and Wiersema, P. H., J. Colloid Interface Sci. 49, 281 (1974).

30. Derjaguin, B. V., Disc. Faraday Soc. 18, 85 (1954).

31. Hogg, R., Healy, T. W., and Fuerstenau, D. W., Trans. Faraday Soc. 62, 1638 (1966).

32. Matthews, B. A., and Rhodes, C. T., J. Colloid Interface Sci. 32, 332 (1970).

33. Wiese, G. R., and Healy, T. W., Trans. Faraday Soc. 66, 490 (1970).

34. Harding, R. D., J. Colloid Interface Sci. 40, 164 (1972).

35. James, R. O., Homola, A., and Healy, T. W., J. Chem. Soc. Faraday Trans. I 73, 1436 (1977).

36. Sasaki, H., Matijević, E., and Barouch, E., J. Colloid Interface Sci. 76, 319 (1980).

37. Adler, P. M., J. Colloid Interface Sci. 84, 461 (1981).

38. Adler, P. M., J. Colloid Interface Sci. 84, 475 (1981).

39. Adler, P. M., J. Colloid Interface Sci. 84, 489 (1981).

40. Hahn, A. U., and Vold, R. D., J. Colloid Interface Sci. 51, 133 (1975).

41. Vold, R. D., and Hahn, A. U., in "Colloidal Dispersions and Micellar Behavior" (K. L. Mittal, Ed.), ACS Symposium Series No. 9, pp. 64-75. Amer. Chem. Soc., Washington, 1975.

42. Vold, R. D., Mittal, K. L., and Hahn, A. U., in "Surface and Colloid Science" (E. Matijević, Ed.) Vol. 10, pp. 45-97. Plenum, New York, 1978.

43. Hahn, A. U., and Mittal, K. L., Colloid Polym. Sci. 257, 959 (1979).

44. El-Aasser, M. S., and Robertson, A. A., J. Colloid Interface Sci. 36, 86 (1971).

45. Melville, J. B., Willis, E., and Smith, A. L., J. Chem. Soc. Faraday Trans. I 68, 450 (1972).

46. Smith, E. R., Mitchell, D. J., and Ninham, B. W., $J$. Colloid Interface Sci. 45, 55 (1973).

47. Pashley, R. M., J. Colloid Interface Sci. 80, 153 (1981).

48. Pailthorpe, B. A., and Russel, W. B., J. Colloid Interface Sci. 89, 563 (1982).

49. Christenson, H. K., J. Chem. Soc. Faraday Trans. I 80, 1933 (1984).

50. Rajagopalan, R., "Stochastic Modeling and Experimental Analysis of Particle Transport in Water Filtration," Ph.D. thesis. Syracuse University, 1974.
51. Feke, D. L., "Kinetics of Flow-Induced Coagulation with Weak Brownian Diffusion," Ph.D. thesis. Princeton University, 1981.

52. Hamaker, H. C., Physica 4, 1058 (1937).

53. Ho, N. F. H., and Higuchi, W. I., J. Pharm. Sci. 57, 436 (1968).

54. Gregory, J., Disc. Faraday Soc. 42, 168 (1966).

55. Barouch, E., and Matijević, E., J. Chem. Soc. Faraday Trans. I 81, 1797 (1985).

56. Barouch, E., Matijević, E., Ring, T. A., and Finlan, J. M., J. Colloid Interface Sci. 67, 1 (1978).

57. Hearn, J., Wilkinson, M. C., and Goodall, A. R., $A d v$. Colloid Interface Sci. 14, 173 (1981).

58. Goodwin, J. W., Hearn, J., Ho, C. C., and Ottewill, R. H., Colloid Polym. Sci. 252, 464 (1974).

59. Lichtenbelt, J. W. TH., Ras, H. J. M. C., and Wiersema, P. H., J. Colloid Interface Sci. 46, 522 (1974).

60. Melik, D. H., "Flocculation in Quiescent Media," Ph.D. thesis, University of Michigan, 1984.

61. Kerker, M., "The Scattering of Light and Other Electromagnetic Radiation," (a) p. 446; (b) p. 50, Academic Press, New York, 1969.

62. Benoit, H., Ullman, R., Devries, A. J., and Wippler, C., J. Chim. Phys. 59, 889 (1962).

63. Egusa, S., J. Colloid Interface Sci. 86, 135 (1982).

64. Heller, W., and Tabibian, R. M., J. Colloid Sci. 12, 25 (1957).

65. Walstra, P., Brit. J. Appl. Phys. 16, 1187 (1965).

66. Latimer, P., J. Opt. Soc. Amer. 62, 208 (1972).

67. Deepak, A., and Box, M. A., Appl. Opt. 17, 2900 (1978).

68. Deepak, A., and Box, M. A., Appl. Opt. 17, 3169 (1978).

69. Zeichner, G. R., "Effects of Hydrodynamics and Colloidal Forces on the Coagulation of Dispersions," Ph.D. thesis. Princeton University, 1978.

70. Melik, D. H., and Fogler, H. S., in "Macro- and Microemulsions: Theory and Applications" (D. O. Shah, Ed.), ACS Symposium Series No. 272, pp. 461-470. Amer. Chem. Soc., Washington, 1983.

71. Melik, D. H., and Fogler, H. S., unpublished data.

72. Parsegian, V. A., and Weiss, G. H., J. Colloid Interface Sci. 81, 285 (1981).

73. Duckworth, R. M., and Lips, A., J. Colloid Interface Sci. 64, 311 (1978).

74. Prieve, D. C., and Lin, M. M. J., J. Colloid Interface Sci. 86, 17 (1982).

75. Rajagopalan, R., and Kim, J. S., J. Colloid Interface Sci. 83, 428 (1981).

76. Adamcyzk, Z., Dảbroś, T., Czarnecki, J., and van de Ven, T. G. M., Adv. Colloid Interface Sci. 19, 183 (1983).

77. Weaver, D. W., and Feke, D. L., J. Colloid Interface Sci. 103, 267 (1985).

78. Barouch, E., and Matijević, E., J. Colloid Interface Sic. 105, 552 (1985).

79. Feke, D. L., and Schowalter, W. R., J. Colloid Interface Sci. 106, 203 (1985). 